\title{
Effect of Ethane-1,2-Diamine on Growth of ZnO Nanorods and Cyclohexane Sensing by Current-Voltage Characteristics Investigations
}

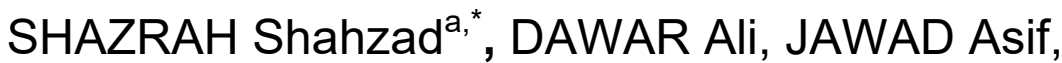 \\ MUHAMMAD Zafar Khan MUHAMMAD Aftab Akram ${ }^{\mathrm{b}}$, SOFIA Javed, \\ Umair Manzoor and MOHAMMAD Mujahid
}

\author{
School of Chemical and Materials Engineering (SCME), \\ National University of Sciences and Technology (NUST), H-12, Islamabad 44000, Pakistan \\ ashazrah_nse03@scme.nust.edu.pk, baftabakram@scme.nust.edu.pk
}

Keywords: Vertically Aligned ZnO Nanorods, Ethane-1,2-diamine, Chemical Bath Deposition, IV characteristics, Cyclohexane.

\begin{abstract}
The growth of vertically aligned $\mathrm{ZnO}$ Nanorods arrays using Zinc Nitrate hexahydrate and Hexamethylene Tetramine (HMTA), by Chemical Bath Deposition on Silicon Wafer was investigated. The growth was conducted under influence of Ethane-1,2-diamine, the amine based enhancer in three different ratios $(1: 0.5,1: 1,1: 1.5)$. The effect different ratios of enhancers on morphology and aspect ratio by Scanning electron microscope (SEM) and crystallinity of the as grown nanorods by X-ray powder diffraction (XRD). Electrical Properties such as current-voltage characteristics were investigated, its correlation to the morphology and aspect ratio of the nanorods in the presence of $100 \mu \mathrm{L}-500 \mu \mathrm{L}$ of Aromatic Compound Cyclohexane and at different applied voltages.
\end{abstract}

\section{Introduction}

Zinc Oxide $(\mathrm{ZnO})$ is a versatile functional direct band gap of $3.37 \mathrm{ev}$ and a large binding energy. 1D $\mathrm{ZnO}$ nanostructures such as nanotubes [1], nanowires [2], nanorods [3], nanobelts [4], tetrapods [5] and nanoribbons [6] stimulate considerable interests for scientific research due to their importance in fundamental physics studies and their potential applications in various fields. There Synthesis routes for $\mathrm{ZnO}$ Nanorods are in a great number and include Chemical bath deposition [7] hydrothermal method [8], Vapor-phase transport [9] Pulsed laser deposition [10]. Solution based synthesis method offer the distinct advantage of being low cost and can be performed at temperatures less than $100^{\circ} \mathrm{C}$ and most importantly the process formulated is easily implemented [11].

The growth of $\mathrm{ZnO}$ Nanorods is carried out in the presence of hexamethylenetetramine (HMTA), which promotes axial growth in the media, there are studies being performed to evaluate and better understand the effect of precursors in the growth of $\mathrm{ZnO}$ Nanorods [12,13]. Along with HMTA, other enhancers have also been studied, which have a pronounced effect on the growth of $\mathrm{ZnO}$ Nanorods [14]. It has been observed that flowerlike structures are formed, when HMTA is used for thermolysis of a zinc complex with Ethane-1,2-diamine [15]. The effect of HMTA has been observed in all these papers and more, effect of temperature and time has been realised as in important parameter for the growth of vertically aligned Nanorods and papers detailing and evaluating the effect of temperature and time can be found in literature [16,17]. HMTA has been realised as to be the driving force for 1 dimensional growth in $\mathrm{ZnO}$ nanorods chemical synthesis routes. It can be referred as driving force with studies detailing the effect of equimolar and nonequimolar concentration effects of HMTA can be found in Literature [18-20]. This serves as motivation for the study, as to amine group based compound will affect the growth of $\mathrm{ZnO}$ Nanorods, while all other conditions like time, temperature, concentration of precursor is kept the same. For this purpose, Ethane 1-2 Diamine is taken into consideration. The effect in three different ratios of precursor (Zinc Nitrate and HMTA) to the enhancer (Ethane 1-2 Diamine), the ratios 1:0.5, $1: 1,1: 1.5$ were studied. 


\section{Experimental Section}

Synthesis of ZnO Seed Layer. The Synthesis of the $\mathrm{ZnO}$ Nanorods is done by following two steps, depositing seed layer and growth on the seeded silicon (100) wafer substrate. The substrates were pre-cleaned in water, acetone and ethanol (30 minutes each) by using bath sonicator, before deposition of seed layer. The Seed layer was coated by using spin coating $10 \mu \mathrm{L}$ of $\mathrm{ZnO}$ seed solution (5mM Zinc Acetate in Absolute Ethanol), the wafer was spun at 3000 RPM for 20 seconds. The Substrates were annealed at $350^{\circ} \mathrm{C}$ for 20 minutes. (Fig. 1)

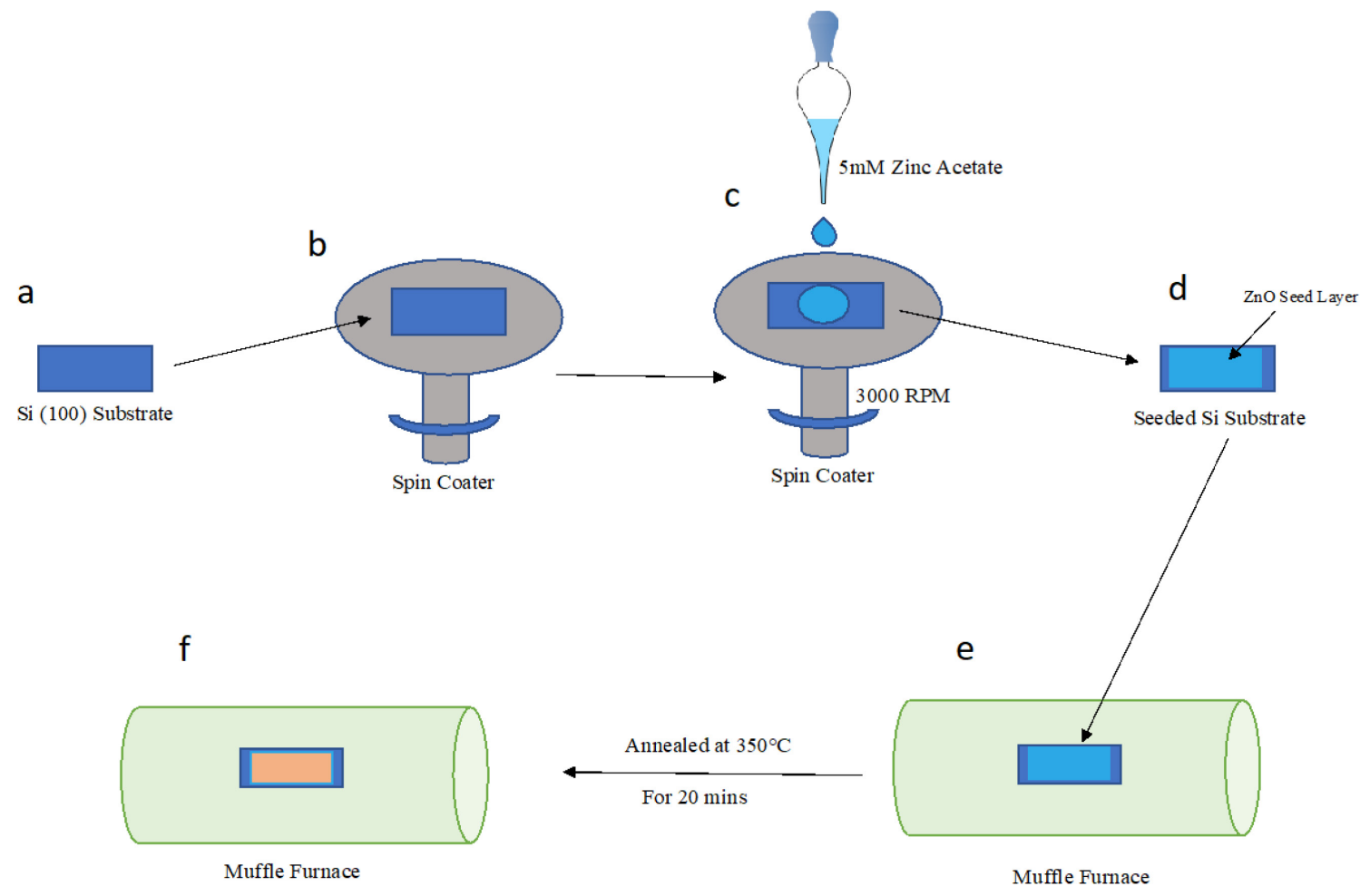

Fig. 1. The process flow for seed layer formation. a) Pre-cleaned Silicon (100) Substrate, b) the Si wafer placed on Spin coater stage with 3000 RPM parameters setting, c) $10 \mu \mathrm{L}$ of $5 \mathrm{mM}$ Zinc Acetate (seeding sol) was dropped on the substrate via micropipette, d) the substrate was allowed to dry in air, e) the sample was placed in muffle furnace and annealed at $350^{\circ} \mathrm{C}$ for $20 \mathrm{mins}$, and f) $\mathrm{ZnO}$ seed layer deposited substrate

Synthesis of ZnO Nanorods. ZnO nanorods were grown using chemical bath deposition, the bath comprises precursors, $0.025 \mathrm{M}$ (or $25 \mathrm{mM}$ ) Zinc Nitrate $\left(\mathrm{ZnNO}_{3}\right)$ and $0.025 \mathrm{M}$ (or $25 \mathrm{mM}$ ) HexamethyleneTetramine (HMTA). In Addition to these precursors the bath solution contains, amine group based enhancer Ethane-1,2-diamine. The enhancer was evaluated based on three different ratios of precursor (Zinc Nitrate and HMTA) to the enhancer, the ratios were 1:0.5, 1:1, 1:1.5 (Zinc Nitrate/HMTA: Enhancer). The seeded substrate was placed in the bath vertically via clamp and the bath was heated to $90^{\circ} \mathrm{C}$ for one hour. The substrate was then further heat treated at $450^{\circ} \mathrm{C}$ for 1 hour. (Fig. 2).

\section{Characterization}

The prepared samples were characterized by using Scanning Electron Microscopy (SEM) (JEOL-JSM- 6490LA) with operating voltage of 10-20 kV, spot size of 35-60, and working distance of $10 \mathrm{~mm}$ and X-Ray Diffractions (XRD) (STOE diffractometer) in $2 \theta$ range of 20-50 degrees was performed to determine the crystalline nature of $\mathrm{ZnO}$ Nanorods. 


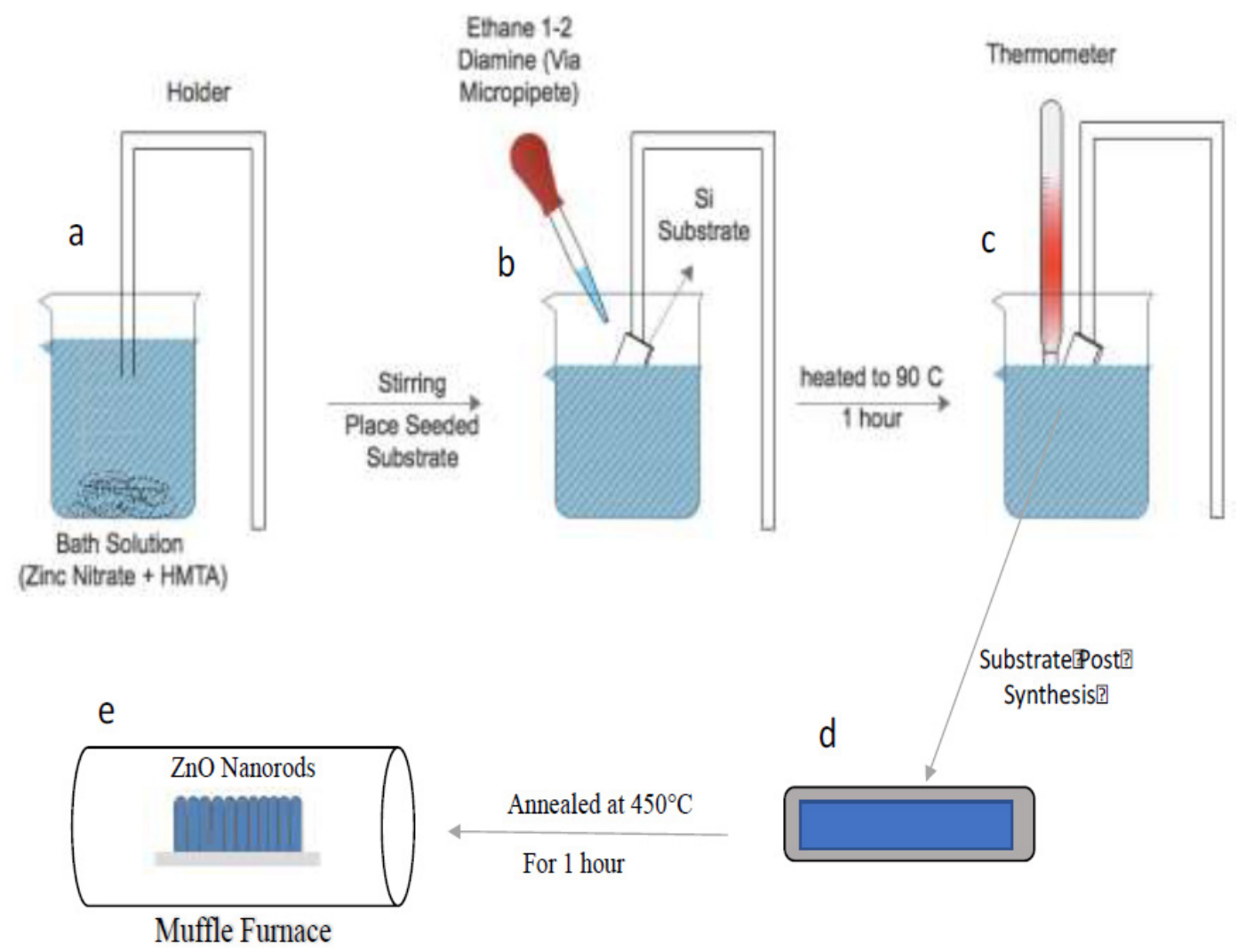

Fig. 2. Synthesis Route for ZnO Nanorods. a) Bath Solution was prepared (25mM of Zinc Nitrate and HMTA), b) Seeded Substrate was placed vertically in the bath and Ethane 1-2 Diamine was added to the bath solution in different ratios, c) the solution was heated to $90^{\circ} \mathrm{C}$ for one hour, d) the substrate was removed from the bath and cleaned with water and ethanol, and e) the substrate was annealed at $450^{\circ} \mathrm{C}$ for 1 hour

I-V Characteristic Investigations (Cyclohexane Sensing). Samples prepared at different ratios were characterized using Electrochemical Workstation (VSP EC-Lab). By this equipment I-V characteristics of these samples were calculated. For Investigation of IV characteristics, Contacts were made using quick drying silver paste. The Cyclohexane concentration was varied between $100,200,300,400$ and $500 \mu \mathrm{L}$. The substrate acts as an electrode and is dipped in water to which the cyclohexane is added in varying concentration. The system assembly is as shown in Fig. 3.

\section{Results and Discussion}

Fig. 4 represent the surface morphology of the as grown Nanorods in various ratios and it can be observed via the SEM images that addition of Ethane 1-2 Diamine, to the bath containing Zinc Nitrate and HMTA, the rods formed have pointed ends, and as ratio is increased the structures become more pointed and lesser is diameter. When the ratio of Ethane 1-2 Diamine is low (Fig. 4a), the rods formed are branched together, few placed horizontally and few vertically aligned. In case of ratio 1:1 (Fig. 4b) the surface coverage observed on the substrate is better as compared to ratio 1:0.5 (low), and it is observed that the diameter of the grown rods has further decreased, with all the rods vertically aligned. At increased ratio (Fig. 4c, ratio 1.5) the rods grown observed have angled rather than vertical alignment observed at ratio 1 . At EDA ratio 0.5 the diameter $\sim 250 \mathrm{~nm}$, but at ratio 1 and 1.5 the average diameter is approximately $\sim 130 \mathrm{~nm}$ and $90 \mathrm{~nm}$ respectively.

As such with increasing the ratio of EDA average diameter and length of the grown 1D structures is affected i.e. decreases. In earlier studies the effect of precursor solution was evaluated on basis of dielectric constant of the solution, with the decrease in dielectric constant the solubility of the inorganic salt decreases as well. With addition of precursors like Ethane 1-2 Diamine, the dielectric constant of the solution changes, which results in changes observed in the morphology as shown in SEM images. 


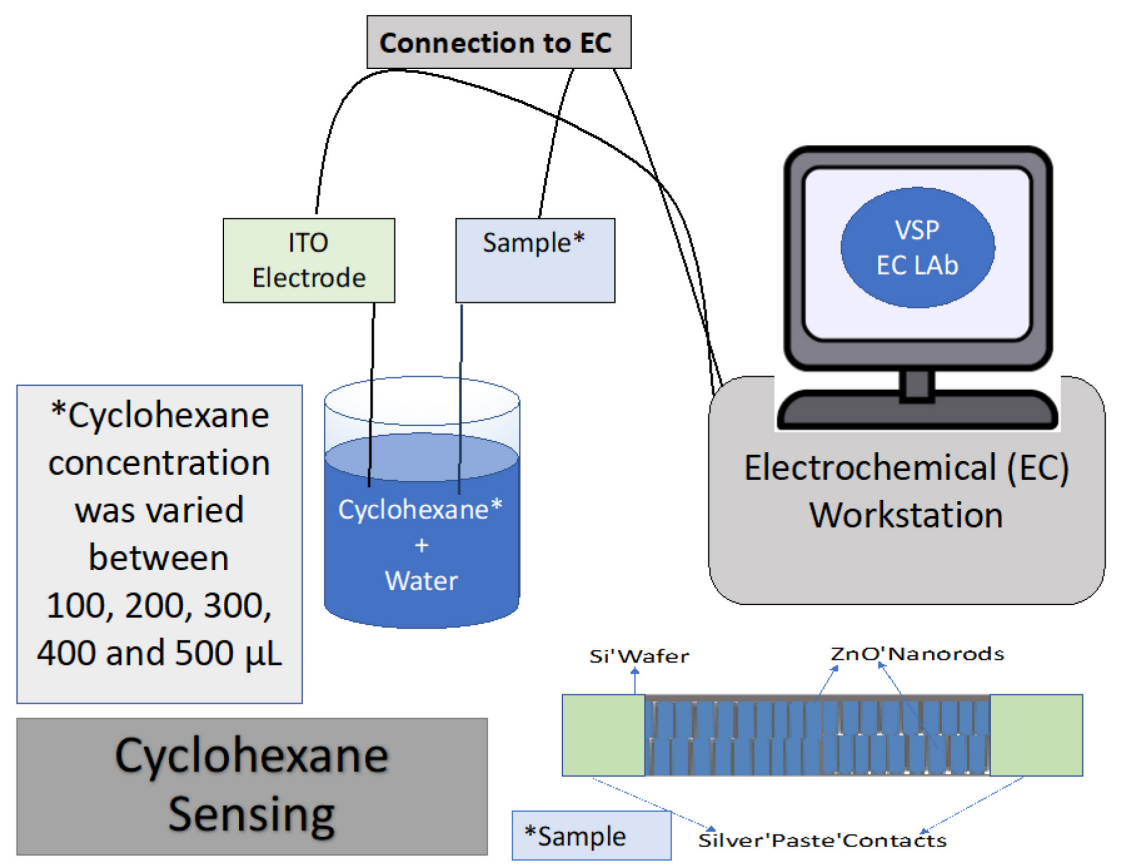

Fig. 3. Schematic of IV Characteristics Investigations
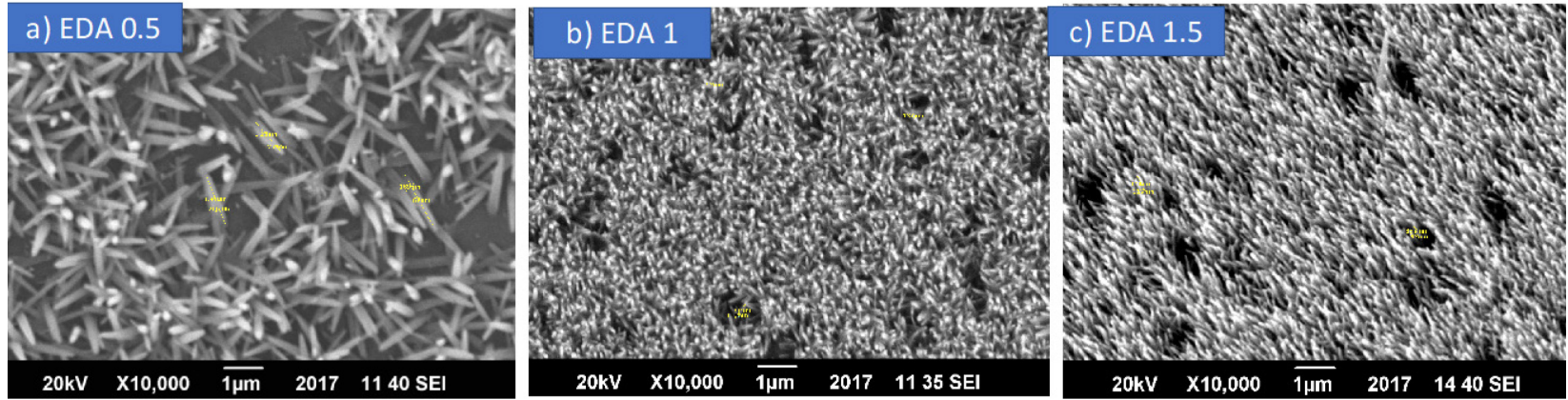

Fig. 4. SEM images of ZnO Nanorods. (Zinc Nitrate/HMTA: EDA [Ethane 1-2 Diamine]) a) 1:0.5 Ratio 25mM Zinc Nitrate, HMTA and 12.5mM EDA), b) 1:1 ratio (25mM Zinc Nitrate, HMTA and EDA) and, c) 1:1.5 (25mM Zinc Nitrate, HMTA and 37.5mM EDA)

Fig. 5(a) shows the XRD pattern observed. The XRD Pattern evidences in favor of $\mathrm{ZnO}$ Nanorods formed (JCPDF file 211486, 030888. The XRD confirms the hexagonal Wurtzite structure of $\mathrm{ZnO}$ Nanostructures formed, single sharp peak corresponding to plane (002) show successful c-axis growth. Few small peaks not corresponding to the $\mathrm{ZnO}$ wurtzite structure are due to presence of Zinc Silicate which is formed when the heat treatment of seed layer is done as it contains $\mathrm{ZnO}$ coated sol over Silicon wafer, the peak is observed because of some uncovered area. The peak shifts towards increasing angles in confirmation to the more pointed ends (needles like end) being formed as successive ratios are increased.

Fig. 5 (b, c, d) show the results observed for changes observed in IV characteristics of the samples with addition of cyclohexane. A trend of deceasing current is observed when the concentration of cyclohexane is increased, the cyclohexane mixed with water causes a change in electrochemical environment of the placed substrate. The change in environment around the dipped substrate manifests in form of decreased current observed. At 1:0.5 ratio (Fig. 5b) the early results with 100 to $200 \mu \mathrm{L}$ and then to $300 \mu \mathrm{L}$ concertation a uniform decrease is observed but at increased concentrations the effect is not that prominent and the decrease becomes small. In case of 1 ratio (Fig. 5c), the difference between current observed from 100 to $200 \mu \mathrm{L}$ is very prominent, but after that the increasing concentration shows negligible changes in current. While for 1.5 ratio of EDA (Fig. 5d) depicts a uniform decrease in current for concentrations 100 to $500 \mu \mathrm{L}$. In case of noncoated samples, i.e. Silicon substrate as it is, the test results show an insulating behavior. 


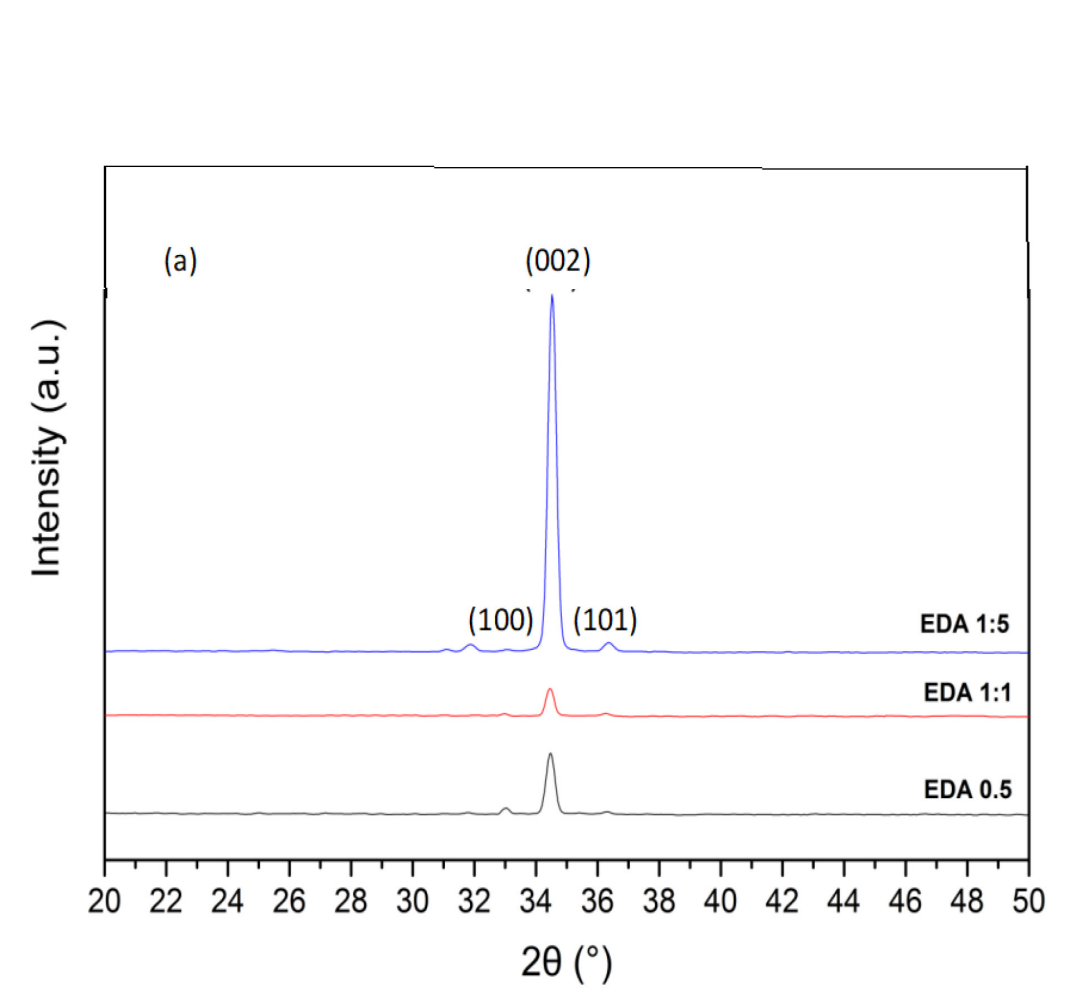

(b)

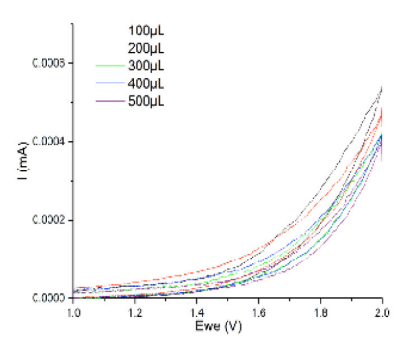

(c)

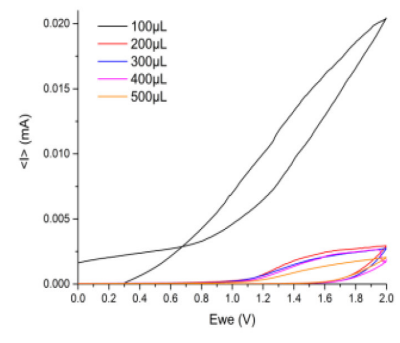

(d)

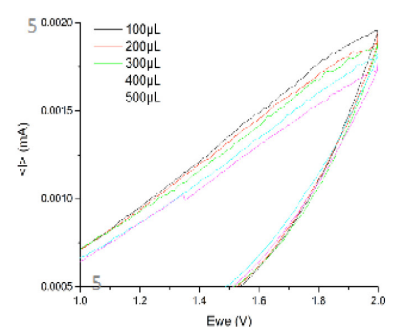

Fig. 5. (a) XRD Pattern observed for $\mathrm{ZnO}$ nanorods. (b) IV characteristics 1:0.5 Ratio, c) IV characteristics $1: 1$ ratio and, d) IV characteristics $1: 1.5$ in presence of $100 \mu \mathrm{L}-500 \mu \mathrm{L}$ cyclohexane

\section{Conclusions}

Growth of $\mathrm{ZnO}$ nanostructures showing different morphology with the addition of Ethane 1-2 Diamine. It can be observed in the SEM images, that with all other variables as constant, addition of EDA in various concentration affecting the system. The length and diameter of the as grown nanorods is different for three ratios, (Fig. 4.). Hence it can be hypothesized that the aspect ratio of the nanorods grown is affected by addition of Ethane 1-2 Diamine. The XRD plots is used to confirm that the samples produced are single crystalline as depicted by one sharp peak (002). The as grown rods show different IV characteristics in presence of cyclohexane, with the 1:5 ratio rods showing a systematic decease in current values with addition of Cyclohexane, showcasing its potential as a sensing material.

\section{Acknowledgements}

We would also like to thank Shayan Naveed, Ramsha Khan and Adeel Riaz for useful discussions. Special mentions for Lab Tech. Shams Ud Din and Lab Tech. Khurram Shahzad for assistance in obtaining SEM and XRD data.

\section{References}

[1] L. Vayssieres, K. Keis, A. Hagfeldt, and S.E. Linquist, Three-Dimensional Array of Highly Oriented Crystalline ZnO Microtubes, Chem.Mater., 13 (2001) 4395-4398.

[2] Y.J.Xing, Y. Song, S. L. Zhang, D. P. Yua, Optical properties of the ZnO nanotubes synthesized via vapor phase growth, Appl. Phy. Lett., 83 (2003) 1689.

[3] Z. W. Pan, Z. R. Dai, Z. L. Wang, Nanobelts of semiconducting oxides, Sci., 291(2001) 19471949. 
[4] W. Z. Wang, B. Q. Zeng, J. Yang, B. Poudel, J. Y. Huang, Aligned Ultralong ZnO Nanobelts and Their Enhanced Field Emission, Adv. Mater., 18 (2006) 3275-3278.

[5] Y . Qiu, S. Y Ang, ZnO Nanotetrapods: Controlled Vapor-Phase Synthesis and Application for Humidity Sensing, Adv. Func. Mater., 17 (2007) 1345-1352.

[6] X. Fan, M. L. Zhang, I. Shafiq, W. J. Zhang, ZnS/ZnO heterojunction nanoribbons, Adv. Mater., 21 (2009) 2393-2396.

[7] N. Boukos, C. Chandrinou, K. Giannakopoulos, G. Pistolis, A. Travlos, Growth of ZnO nanorods by a simple chemical method, Appl. Phy. A, 88 (2007) 35-9.

[8] P. Rai, S. K. Tripathy, Nam-Hee Park, Kwang-Joong O, In-Hwan Lee, Yeon-Tae Yu, Synthesis of violet light emitting single crystalline $\mathrm{ZnO}$ Nanorods by using CTAB-assisted hydrothermal method, J Mater Sci: Mate.r Electron., 20 (2009) 967-971

[9] Y. Sun, G. M. Fuge and M. N. R. Ashfold, Growth of aligned ZnO nanorod arrays by catalystfree pulsed laser deposition methods, Chem. Phys. Lett., 396 (2004) 21-26.

[10] J.Wu and S. C. Liu, Low-temperature growth of well-aligned $\mathrm{ZnO}$ nanorods by chemical vapor deposition, Adv. Mater., 14 (2002) 215-218

[11] S. Xu, Z. L. Wang, One-Dimensional ZnO Nanostructures: Solution Growth and Functional Properties. Nano. Res., 4 (2011) 1013-1098.

[12] M. Wang, C. Xing, K. Cao, K, L. Meng, J. Liu, Alignment-Controlled Hydrothermal Growth of Well Aligned ZnO Nanorod Arrays. J. Phys. Chem. Solids., 75 (2014) 808-817.

[13] V. Strano, V, R. G. Urso, R. G, M. Scuderi, K.O. Iwu, F. Simone, E. Ciliberto, C. Spinella, S. Mirabella, Double Role of HMTA in ZnO Nanorods Grown by Chemical Bath Deposition. J. Phys. Chem. C, 118 (2014) 28189-28195.

[14] Y. Zhou, W. Wu, G. Hu, H. Wu, S. Cui, Hydrothermal synthesis of ZnO nanorod arrays with the addition of polyethyleneimine, Mat. Res. Bull., 43 (2008) 2113-2118

[15] X. Gao, X. Li, W. Yu, Flowerlike ZnO Nanostructures via Hexamethylenetetramine-Assisted Thermolysis of Zinc-Ethylenediamine Complex, J. Phys. Chem. B, 109 (2005) 1155-1161.

[16] T.S. Senthil, A-Young Kim, N. Muthukumarasamy, M. Kang, Effect of bath temperature on the performance of $\mathrm{ZnO}$ nanorod-based thin film solar cells. J. Nanopart. Res., 15 (2013) 19261932.

[17] Y. Qua, X. Huanga, Y. Lia, G.Lina, B. Guoa, D. Songb, Q. Chenga. Size Controllable of ZnO Nanorods on Si Substrates. Superlattic. Microstruct., 101 (2017) 469-479.

[18] R. Parize, J. D. Garnier, O. Chaix- Pluchery, C. Verrier, E. Appert, and V. Consonni. "Effects of HMTA on the Nucleation and Radial Growth of $\mathrm{ZnO}$ Nanowires by Chemical Bath Deposition.” J. Phys. Chem. C., 27 (2016) 327-342.

[19] A. Sugunan, H.C. Warad, M. Boman, J. Dutta. Zinc Oxide Nanowires in Chemical Bath on Seeded Substrates: Role of Hexamine. J. Sol-Gel Sci. Techn., 39 (2016) 49-56.

[20] K. M. McPeak, T. P. Le, N. G. Britton, Z. S. Nickolov, Y. A. Elabd, J. B. Baxter, Chemical Bath Deposition of $\mathrm{ZnO}$ Nanowires at Near-Neutral $\mathrm{pH}$ Conditions Without Hexamethylenetetramine (HMTA): Understanding the Role of HMTA in ZnO Nanowire Growth, Langmuir, 27 (2011) 3672-3677. 